\title{
Satralizumab: First Approval
}

\author{
Young-A Heo ${ }^{1}$ \\ Published online: 14 August 2020 \\ (c) Springer Nature 2020, corrected publication 2020
}

\begin{abstract}
Satralizumab (Enspryng ${ }^{\circledR}$ ), a humanized anti-interleukin-6 (IL-6) receptor monoclonal recycling antibody, has been developed by Chugai Pharmaceutical and Roche for the treatment of neuromyelitis optica spectrum disorder (NMOSD). In June 2020, based on positive results from two pivotal phase III trials, subcutaneous satralizumab received its first global approval in Canada for the treatment of NMOSD in adults and children aged $\geq 12$ years who are aquaporin 4 water channel autoantibody (AQP4-IgG) seropositive. Satralizumab was subsequently approved in Japan, Switzerland and the USA. Satralizumab is under regulatory review in the EU, and is undergoing clinical development in several countries worldwide. This article summarizes the milestones in the development of satralizumab leading to this first approval for the treatment of NMOSD.
\end{abstract}

\section{Satralizumab (Enspryng ${ }^{\oplus}$ ): Key points}

A humanized anti-IL-6 receptor monoclonal recycling antibody being developed by Chugai Pharmaceutical and Roche for the treatment of NMOSD

Received its first approval on 1 June 2020 in Canada Approved for use in Canada as a monotherapy or as a combination therapy with immunosuppressant in adults and children aged $\geq 12$ years with NMOSD who are AQP4-IgG seropositive
Enhanced material for this AdisInsight Report can be found at https://doi.org/10.6084/m9.figshare.12762386.

This profile has been extracted and modified from the AdisInsight database. AdisInsight tracks drug development worldwide through the entire development process, from discovery, through preclinical and clinical studies to market launch and beyond.

Young-A Heo

dru@adis.com

1 Springer Nature, Private Bag 65901, Mairangi Bay, Auckland 0754, New Zealand

\section{Introduction}

Neuromyelitis optica spectrum disorder (NMOSD) is a rare autoimmune disease of the central nervous system (CNS) that is characterized by inflammatory and demyelinating lesions in optic nerve, spinal cord brainstem and cerebrum, which can lead to progressive impairment of vision and motor functions [1-4]. The primary goal of NMOSD treatment is to reduce the risk of irreversible neurological impairment by preventing relapse and reducing the severity of attacks [5, 6]. Recent research suggested that interleukin-6 (IL-6) has an important role in the immunopathogenesis of NMOSD [1, 2]. IL-6 signalling triggers an inflammatory cascade that is thought to lead to differentiation of $\mathrm{T}$ cells into proinflammatory $\mathrm{TH} 17$ cells, differentiation of $\mathrm{B}$ cells into plasmablasts that produce aquaporin 4 water channel autoantibodies (AQP4-IgG), a diagnostic serum marker that is found in $\approx 80 \%$ of patients with NMOSD, and an increase in blood-brain barrier (BBB) permeability, allowing penetration of antibodies and proinflammatory cells into the CNS [2,7-10].

Satralizumab (Enspryng ${ }^{\circledR}$ ), a humanized anti-IL-6 receptor monoclonal antibody designed using recycling antibody technology $^{\mathrm{TM}}$, has been developed by Chugai Pharmaceutical and Roche for the treatment of NMOSD [11]. Based on positive results from two pivotal phase III trials, satralizumab received its first global approval under priority review in Canada on 1 June 2020 for the treatment of NMOSD as monotherapy or as combination therapy with immunosuppressant in adults and children aged $\geq 12$ years who are AQP4IgG seropositive [11]. On 29 June 2020, satralizumab was 


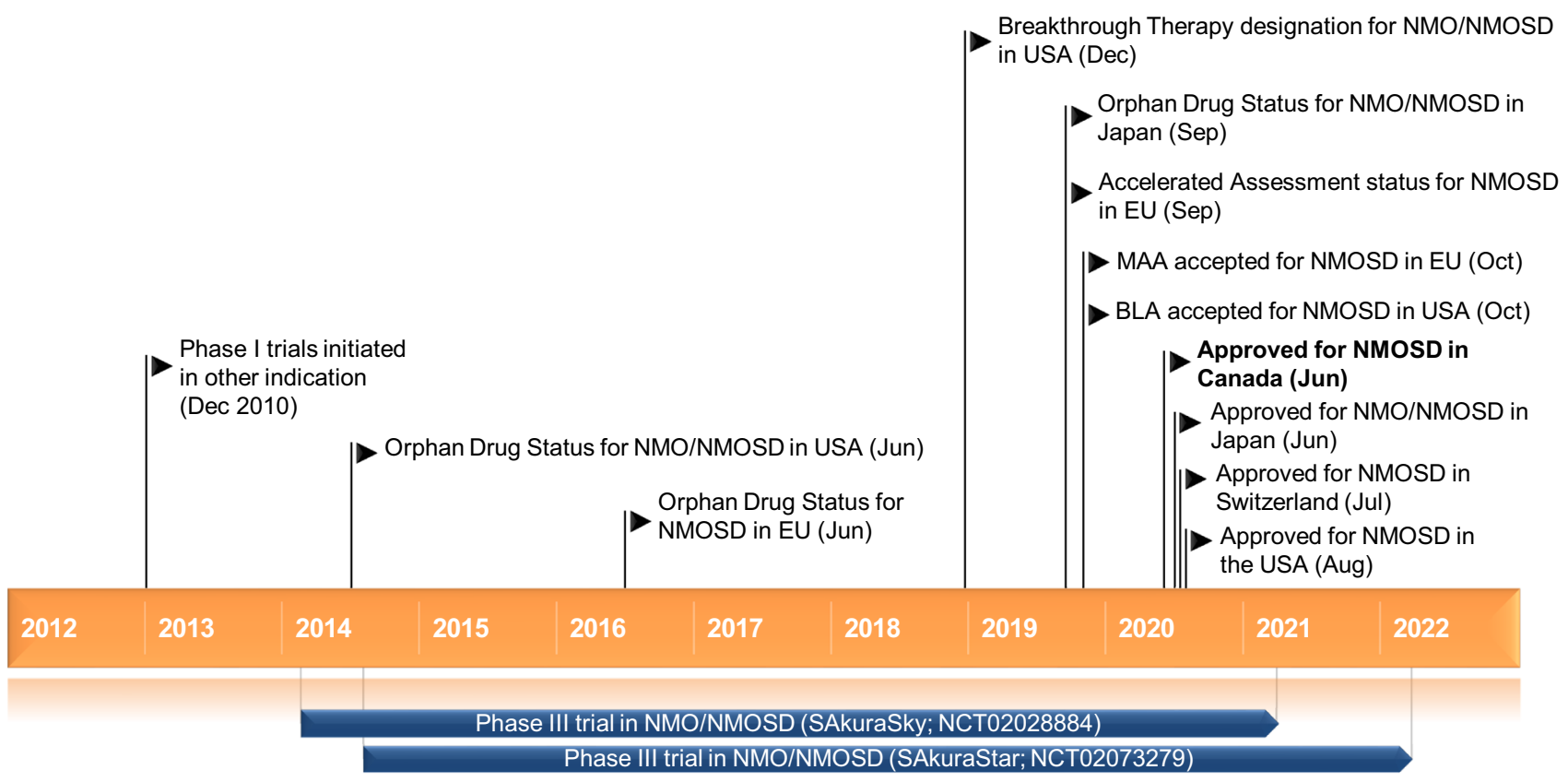

Key milestones in the development of satralizumab BLA Biologics License Application, MAA Marketing Authorization Application, NMO neuromyelitis optica, $N M O S D$ neuromyelitis optica spectrum disorder

subsequently approved in Japan for the prevention of relapses of NMOSD, including neuromyelitis optica (NMO), in adults and children who are AQP4-IgG seropositive [12]. On 13 July 2020, satralizumab was approved in Switzerland for the treatment of NMOSD as monotherapy or as combination therapy with immunosuppressant in adults and adolescents who are AQP4-IgG seropositive [13]. On 17 August 2020, satralizumab was approved in the USA for the treatment of NMOSD in adult patients who are AQP4 antibody positive $[14,15]$.

Satralizumab is available as a single-use, prefilled syringe containing $120 \mathrm{mg} / \mathrm{mL}$ satralizumab for subcutaneous injection and the recommended dosage is $120 \mathrm{mg}$ at week 0,2 and 4 as loading doses, followed by a maintenance dose of 120 mg every 4 weeks $[13,15-17]$. The first subcutaneous injection of satralizumab should be administered under supervision of a health practitioner. During satralizumab treatment, liver enzyme levels should be monitored every 4 weeks for the first 3 months, followed by every 3 months for 1 year; neutrophil counts should be monitored 4-8 weeks after treatment initiation and as clinically indicated thereafter [13, 15-17].

Subcutaneous satralizumab is under regulatory review in the EU $[18,19]$, and is undergoing clinical development in several countries worldwide. In Japan, phase I clinical development was conducted for the treatment of rheumatoid arthritis; however, no recent development reports have been identified.

\subsection{Company Agreements}

In June 2016, Chugai Pharmaceutical and Roche entered into an exclusive worldwide license agreement under which the latter was granted the worldwide rights for the development and marketing of satralizumab, with the exception of Japan and Taiwan [20,21]. Under the terms of the agreement, Chugai is entitled to receive an upfront fee, milestone and royalty payments from Roche, while being responsible for continued product manufacturing and supply of satralizumab [20].

\section{Scientific Summary}

\subsection{Pharmacodynamics}

Satralizumab is a humanized immunoglobulin G2 monoclonal antibody produced in Chinese hamster ovary cells using recombinant DNA technology [16]. The exact mechanisms of action of satralizumab in treating NMOSD is currently unknown, but is thought to target multiple aspects that contribute to the disease in patients with NMOSD by binding to membrane-bound and soluble IL-6 receptors, thereby blocking IL-6 signalling pathways $[6,10,16]$. Through inhibition of downstream IL-6 signalling pathways, satralizumab is thought to reduce inflammation and IL-6 mediated autoimmune $\mathrm{T}$ - and $\mathrm{B}$-cell activation, preventing differentiation of B cells into AQP4-IgG-secreting plasmablasts [2, 10]. Satralizumab utilizes a novel recycling antibody technology which allows satralizumab to dissociate from IL-6 receptor in a pH-dependent manner, thereby extending the duration of circulation in the body [2]. After uptake by the cell, the 
antibody-antigen complex is transported to the endosome $[2,22,23]$. In the acidic environment of the late endosomal compartment ( $\mathrm{pH}$ 5.5-6.0), satralizumab dissociates from the IL- 6 receptor and is transported back to the plasma membrane via the recycling endosomal pathway by binding to the neonatal $\mathrm{Fc}$ receptor $(\mathrm{FcRn})$, leading to release of free satralizumab back into plasma, ready to bind another IL-6 receptor [2, 22, 23]. The drug's binding affinity to IL-6 receptor is maintained in plasma ( $\mathrm{pH} 7.4)[2,22]$. Satralizumab has $\approx 4$-fold higher affinity to IL-6 receptor than tocilizumab [6].

In vitro, satralizumab reduced $\mathrm{NMO}$-induced $\mathrm{BBB}$ dysfunction [24]. Moreover, subcutaneous satralizumab significantly inhibited IL-6 receptor signalling for four weeks, with marked, sustained increases in soluble IL-6 receptor levels observed in Japanese and Caucasian healthy volunteers $(n=72)$ and patients with rheumatoid arthritis $(n=33)$ or with NMOSD $(n=104)$ [25]. In NMOSD patients who received subcutaneous satralizumab $120 \mathrm{mg}$ at week 0, 2, 4 (loading regimen) and once every 4 weeks thereafter (maintenance regimen), the predicted median IL-6 receptor occupancy was maintained at $>95 \%$ over the satralizumab 4 -week dosing interval [25-27]. Decreases in C-reactive protein, fibrinogen and complement (C3, C4 and CH50) were also observed with satralizumab treatment [16].

\subsection{Pharmacokinetics}

In healthy volunteers, subcutaneous satralizumab exhibits non-linear pharmacokinetics across the single dose range of 30-240 mg [28].
Following subcutaneous administration of satralizumab at the recommended dosage, the absorption half-life and bioavailability is $\approx 3$ days and $78.5 \%$, respectively, with steady state reached 8 weeks after the first administration, according to a population pharmacokinetic analysis in 154 patients with NMO or NMOSD [16]. Satralizumab is distributed in a biphasic manner where the estimated central and peripheral volume of distributions for a typical $60 \mathrm{~kg}$ patient is $3.46 \mathrm{~L}$ and 2.07 $\mathrm{L}$, respectively. Although the metabolism of satralizumab has not been characterized, the drug is expected to mainly undergo catabolism in the same manner as endogenous IgG. Satralizumab exhibits concentration-dependent, linear and target-mediated (Michaelis-Menten) elimination characteristics, with the estimated linear clearance and associated elimination half-life being $0.0679 \mathrm{~L} /$ day and $\approx 30$ days, respectively [16].

The pharmacokinetics of satralizumab are not affected by age, gender and race [16]. No formal studies of the effect of hepatic or renal impairment on the pharmacokinetics of satralizumab have been conducted. Higher bodyweight and the presence of anti-drug antibodies (ADAs) appeared to have a significant impact on satralizumab exposure; however, based on the results of exposure-response relationship analyses, no dosage adjustment is required [16].

There are no formal drug interaction studies of satralizumab [16]. Since the expression of CYP450 enzymes is suppressed by overproduction of IL-6 in vitro and in vivo, caution should be exercised when satralizumab is initiated or discontinued in patients concomitantly receiving CYP3A4, CYP1A2, CYP2C9 or CYP2C19 substrates, particularly those with a narrow therapeutic index (e.g. warfarin, carbamazepine, phenytoin and theophylline) and dosage can be adjusted if needed [16].

\begin{tabular}{ll} 
Alternative names & ENSPRYNG; RG-6168; SA-237 \\
Class & Anti-inflammatories; Antirheumatics; Eye disorder therapies; Monoclonal antibodies \\
\hline Mechanism of Action & Interleukin 6 receptor antagonists \\
\hline $\begin{array}{l}\text { Route of Administration } \\
\text { Pharmacodynamics }\end{array}$ & Subcutaneous \\
\hline Pharmacokinetics & Inhibits interleukin-6 signalling pathways \\
& $\begin{array}{c}\text { Non-linear pharmacokinetics; steady state reached } 8 \text { weeks after first administration; estimated } \\
\text { mean linear clearance } 0.0679 \text { L/day and the associated elimination half-life } \approx 30 \text { days }\end{array}$ \\
\hline Most frequent adverse events & Headache, arthralgia and injection related reactions \\
\hline ATC codes & L04A-C19 (Satralizumab); M01A (Antiinflammatory and Antirheumatic Products, Non-ster- \\
\hline WHO ATC code & oids) \\
\hline EphMRA ATC code & L4C (Interleukin Inhibitors); M1A (Anti-Rheumatics, Non-Steroidal) \\
Chemical Name & Immunoglobulin G2, anti-(human interleukin 6 receptor) (human-mus musculus monoclonal \\
& SA237 heavy chain), disulfide with human-mus musculus monoclonal SA237 light chain, \\
dimer
\end{tabular}




\subsection{Therapeutic Trials}

The efficacy and safety of satralizumab was evaluated in two pivotal, multinational, randomized, double-blind, placebocontrolled phase III trials, SAkuraStar (NCT02073279) and SAkuraSky (NCT02028884), in patients with NMO or NMOSD, including both AQP4-IgG seropositive and seronegative patients (capped at 30\%) [26, 27]. In SAkuraStar ( $n$ $=95)$ and SAkuraSky $(n=83)$, patients were randomized to receive subcutaneous satralizumab $120 \mathrm{mg}$ or matching placebo at week 0,2 and 4, then once every 4 weeks; satralizumab was administered as a monotherapy (SAkuraStar [26]) or as an add-on therapy to baseline immunosuppressant therapy (SAkuraSky [27]). In SAkuraSky, baseline immunosuppressant therapy included azathioprine (AZA), mycophenolate mofetil (MMF) or oral corticosteroids at stable doses, with adolescent patients permitted to receive a combination of AZA and oral corticosteroids, or MMF and oral corticosteroids [27]. Patients experiencing a protocol-defined relapse (PDR) or those completing the double-blind period of the study were eligible to enter an open-label extension period, during which all patients received unblinded long-term treatment with satralizumab [26, 27].

During the double-blind period, satralizumab was effective in reducing the risk of an adjudicated NMOSD relapse whether it was administered as a monotherapy or as an addon therapy to baseline immunosuppressant therapy [26, 27]. In SAkuraStar (median treatment duration: 92.3 weeks with satralizumab vs 54.6 weeks with placebo), the proportion of patients experiencing a PDR was significantly $(p=0.018)$ lower in the satralizumab monotherapy group than in the placebo group (30\% vs 50\%), with satralizumab reducing the risk of relapse by 55\% [hazard ratio (HR) 0.45, 95\% CI 0.23-0.89; primary endpoint in the intention-to-treat population, encompassing both AQP4-IgG seropositive and seronegative subgroups] [26]. The proportion of relapse-free patients in the satralizumab and placebo groups was $76 \%$ versus $62 \%$ at week $48,72 \%$ versus $51 \%$ at week 96 and $63 \%$ versus $34 \%$ at week 144 , respectively [26]. Similarly, in SAkuraSky (median treatment duration: 107.4 weeks with satralizumab vs 32.5 weeks with placebo), a significantly
( $p=0.02)$ lower proportion of patients receiving add-on satralizumab experienced a PDR than those receiving placebo ( $20 \%$ vs $43 \%$ ), with satralizumab reducing the risk of relapse by $62 \%$ (HR $0.38,95 \%$ CI $0.16-0.88$; primary endpoint in the intention-to-treat population, encompassing both AQP4-IgG seropositive and seronegative subgroups) [27]. The proportion of relapse-free patients in the satralizumab and placebo groups was $89 \%$ versus $66 \%$ at week 48 , $78 \%$ versus $59 \%$ at week 96 and $74 \%$ versus $49 \%$ at week 144, respectively [27]. There was no significant difference between satralizumab and placebo groups in the predefined key secondary outcomes, change in Visual Analogue Scale pain score and the Functional Assessment of Chronic Illness Therapy (FACIT) fatigue score from baseline to week $24[26,27]$.

In a prespecified subgroup analysis, relative to placebo, satralizumab was associated with a significantly lower risk of relapse (based on $95 \%$ CIs) in patients who were AQP4-IgG seropositive (SAkuraStar: HR 0.26, 95\% CI 0.11-0.63; SAkuraSky: HR 0.21, 95\% CI 0.06-0.75); there was insufficient evidence (based on 95\% CIs) to indicate that satralizumab significantly lowered the risk of NMOSD relapse compared with placebo in patients who were AQP4IgG seronegative [26, 27]. In the AQP4-IgG seropositive subgroup, the proportion of relapse-free patients receiving satralizumab or placebo was $83 \%$ versus $55 \%$ at week 48 , $77 \%$ versus $41 \%$ at week 96 and $77 \%$ versus $41 \%$ at week 144 in SAkuraStar [26] and it was $92 \%$ versus $60 \%$ at week $48,92 \%$ versus $53 \%$ at week 96 and $85 \%$ versus $53 \%$ at week 144 in SAkuraSky [27]. Furthermore, the treatment benefit of satralizumab over placebo was consistently seen regardless of age (adolescents aged 13-17 years [29]) or geographical region (Asian region [30]).

The results of a pooled analysis of data from SAkuraStar and SAkuraSky were consistent to those observed in the individual trials $[31,32]$. In the pooled analysis, satralizumab reduced the risk of PDR by 58\% compared with placebo (HR 0.42, 95\% CI 0.25-0.71) and the HR for this endpoint in the AQP4-IgG seropositive group was 0.25 (95\% CI 0.12-0.50) [31, 32].

Key clinical trials of satralizumab

\begin{tabular}{lllllll} 
Drug(s) & Indication & Phase & Status & Location(s) & Identifier & Sponsors \\
\hline $\begin{array}{c}\text { Satralizumab, } \\
\text { placebo }\end{array}$ & NMO/NMOSD & Phase III & $\begin{array}{c}\text { Active, no } \\
\text { longer } \\
\text { recruiting }\end{array}$ & Multinational & $\begin{array}{c}\text { NCT02073279, BN40900, } \\
\text { SA309JG, SAkuraStar }\end{array}$ & $\begin{array}{c}\text { Chugai Pharmaceutical, } \\
\text { Roche }\end{array}$ \\
$\begin{array}{c}\text { Satralizumab, } \\
\text { placebo }\end{array}$ & NMO/NMOSD & Phase III & $\begin{array}{c}\text { Active, no } \\
\text { longer } \\
\text { recruiting }\end{array}$ & Multinational & $\begin{array}{c}\text { NCT02028884, BN40898, } \\
\text { Chugai Pharmaceutical, } \\
\text { Roche }\end{array}$ \\
\hline
\end{tabular}




\subsection{Adverse Events}

Satralizumab was generally well tolerated when administered as a monotherapy or as an add-on therapy to immunosuppressant therapy in patients with NMOSD, based on results from the phase III SAkuraStar and SAkuraSky trials $[26,27,33]$.

The most commonly reported adverse events (AEs) with satralizumab included headache, arthralgia and injection related reactions (IRRs) [16]. In 104 satralizumab recipients in SAkuraStar and SAkuraSky, serious treatment-related AEs, including infections, occurred in $2.9 \%$ of patients and AEs resulted in dose interruptions in $22.1 \%$ of patients and treatment discontinuation in $3.8 \%$ patients; these rates were comparable to those seen in placebo recipients [16]. There were no deaths or anaphylactic reactions reported in SAkuraStar and SAkuraSky $[26,27]$.

In the pooled analysis of data from SAkuraStar and SAkuraSky, the rate of infection in patients receiving satralizumab $(n=104)$ or placebo $(n=74)$ was 113.04 and 154.85 events/100 patient-years (PY), respectively; satralizumab was not associated with an increased risk of opportunistic infections [33]. Satralizumab treatment should not be initiated in patients with active infections and if a patient develops an active infection during the treatment, satralizumab should be interrupted until the infection is controlled [13, 15-17].

Although the rate of IRRs was higher with satralizumab than with placebo (18.58 vs 8.99 events/100 PY), reported IRRs were mostly of mild to moderate severity and none required treatment discontinuation [16, 33]; IRRs mostly occurred within $24 \mathrm{~h}$ after injections [16]. Laboratory abnormalities (e.g. decreases in neutrophil and platelet counts, increases in liver enzymes and total cholesterol) have been reported with satralizumab; however, the majority of the cases were transient and/or resolved without dose interruption [16]. Neutrophil counts and liver enzyme levels should be monitored during satralizumab treatment [13, 15-17].

Longer term, the nature and frequency of AEs with satralizumab during the open-label extension periods in SAkuraStar and SAkuraSky were consistent to those observed during the double-blind periods in these two trials; no new safety signals were identified [34].

As with all therapeutic proteins, satralizumab has a potential for immunogenicity. In SAkuraStar and SAkuraSky, ADAs were detected in $71 \%$ and $41 \%$ of satralizumab recipients, respectively [16]. The presence of ADAs was not associated with changes in pharmacodynamics, efficacy and safety of satralizumab [16].

\subsection{Ongoing Clinical Trials}

The open-label extension periods of phase III SAkuraStar (NCT02073279) and SAkuraSky (NCT02028884) trials are currently ongoing.

\section{Current Status}

Satralizumab received its first approval on 1 June 2020 in Canada for the treatment of NMOSD in adults and children aged $\geq 12$ years who are AQP4-IgG seropositive [11]. Subsequently, satralizumab was approved in Japan on 29 June 2020 , for the prevention of relapses of NMOSD, including NMO, in adults and children who are AQP4-IgG seropositive [12]. On 13 July 2020, satralizumab received approval in Switzerland for the treatment of NMOSD as monotherapy or as combination therapy with immunosuppressant in adults and adolescents who are AQP4-IgG seropositive [13]. On 17 August 2020, satralizumab was approved in the USA for the treatment of NMOSD in adult patients who are AQP4 antibody positive $[14,15]$.

Acknowledgements During the peer review process, the manufacturer of satralizumab was also offered an opportunity to review this article. Changes resulting from comments received were made on the basis of scientific and editorial merit.

\section{Declarations}

Funding The preparation of this review was not supported by any external funding.

Conflict of interest Young-A Heo is a salaried employee of Adis International Ltd/Springer Nature, is responsible for the article content and declares no relevant conflicts of interest.

Ethics approval, Consent to participate and consent for publication, Availability of data and material, Code availability Not applicable.

Open Access This article is licensed under a Creative Commons Attribution-NonCommercial 4.0 International License, which permits any non-commercial use, sharing, adaptation, distribution and reproduction in any medium or format, as long as you give appropriate credit to the original author(s) and the source, provide a link to the Creative Commons licence, and indicate if changes were made. The images or other third party material in this article are included in the article's Creative Commons licence, unless indicated otherwise in a credit line to the material. If material is not included in the article's Creative Commons licence and your intended use is not permitted by statutory regulation or exceeds the permitted use, you will need to obtain permission directly from the copyright holder. To view a copy of this licence, visit http://creativecommons.org/licenses/by-nc/4.0/. 


\section{References}

1. Xue T, Yang Y, Lu Q, et al. Efficacy and safety of monoclonal antibody therapy in neuromyelitis optica spectrum disorders: evidence from randomized controlled trials. Mult Scler Relat Disord. 2020. https://doi.org/10.1016/j.msard.2020.102166.

2. Collongues N, Ayme-Dietrich E, Monassier L, et al. Pharmacotherapy for neuromyelitis optica spectrum disorders: current management and future options. Drugs. 2019;79(2):125-42.

3. Kessler RA, Mealy MA, Levy M. Treatment of neuromyelitis optica spectrum disorder: acute, preventive, and symptomatic. Curr Treat Options Neurol. 2016;18(1):2.

4. Finke C, Zimmermann H, Pache F, et al. Association of visual impairment in neuromyelitis optica spectrum disorder with visual network reorganization. JAMA Neurol. 2018;75(3):296-303.

5. Araki M, Yamamura T. Neuromyelitis optica spectrum disorders: emerging therapies. Clin Exp Neuroimmunol. 2017;8(2):107-16.

6. Papadopoulos MC, Bennett JL, Verkman AS. Treatment of neuromyelitis optica: state-of-the-art and emerging therapies. Nat Rev Neurol. 2014;10(9):493-506.

7. Kawano MM, Mihara K, Huang N, et al. Differentiation of early plasma cells on bone marrow stromal cells requires interleukin-6 for escaping from apoptosis. Blood. 1995;85(2):487-94.

8. Kimura A, Kishimoto T. IL-6: regulator of Treg/Th17 balance. Eur J Immunol. 2010;40(7):1830-5.

9. Yoshizaki K, Nakagawa T, Fukunaga K, et al. Isolation and characterization of $\mathrm{B}$ cell differentiation factor (BCDF) secreted from a human B lymphoblastoid cell line. J Immunol. 1984;132(6):2948-54.

10. Chihara N, Aranami T, Sato W, et al. Interleukin 6 signaling promotes anti-aquaporin 4 autoantibody production from plasmablasts in neuromyelitis optica. Proc Natl Acad Sci USA. 2011;108(9):3701-6.

11. Roche. Roche's Enspryng® (satralizumab) approved in Japan for adults and children with neuromyelitis optica spectrum disorder [media release]. 29 Jun 2020.

12. Chugai Pharmaceutical. Chugai's Enspryng (satralizumab) subcutaneous injection $120 \mathrm{mg}$ syringe approved in Japan for neuromyelitis optica spectrum disorder [media release]. 29 Jun 2020.

13. Roche Pharma (Schweiz) AG. Enspryng®: information for healthcare professionals. 2020. https://www.swissmedicinfo. $\mathrm{ch} /$ ?Lang=EN. Accessed 3 Aug 2020.

14. US Food and Drug Administration. FDA approves treatment for rare disease affecting optic nerves, spinal cord [media release] 17 Aug 2020. https://www.fda.gov/

15. ENSPRYNG ${ }^{\mathrm{TM}}$ (satralizumab-mwge): US prescribing information. https://www.gene.com/download/pdf/enspryng_prescribin g.pdf. Accessed 18 Aug 2020

16. Hoffmann-La Roche Limited. ${ }^{\text {Pr}}$ Enspryng $®$ satralizumab injection solution, $120 \mathrm{mg} / \mathrm{mL}$, subcutaneous: Canadian prescribing information. 2020. https://www.rochecanada.com/. Accessed $1 \mathrm{Jul}$ 2020.

17. Chugai Pharmaceutical. Enspryng (satralizumab) subcutaneous injection 120mg syringe: Japanese prescribing information. 2020. https://www.pmda.go.jp/. Accessed 1 Jul 2020.

18. Roche. Roches marketing applications for satralizumab in neuromyelitis optica spectrum disorder accepted for review by EMA and FDA [media release]. 30 Oct 2019.
19. Chugai Pharmaceutical. EMA and FDA accept marketing applications for Chugai's satralizumab in neuromyelitis optica spectrum disorder (NMOSD) [media release]. 30 Oct 2019.

20. Chugai Pharmaceutical. Chugai announces license agreement for recycling antibody SA237 [media release]. 1 Jun 2016.

21. Chugai Pharmaceutical. Annual report 2019 (integrated report). 2019. https://www.chugai-pharm.co.jp/english/ir/reports_downl oads/annual_reports.html. Accessed 4 Aug 2020.

22. Igawa $\mathrm{T}$, Ishii $\mathrm{S}$, Tachibana $\mathrm{T}$, et al. Antibody recycling by engineered $\mathrm{pH}$-dependent antigen binding improves the duration of antigen neutralization. Nat Biotechnol. 2010;28(11):1203-7.

23. Chugai Pharmaceutical. SMART-Ig® (recycling antibody®). 2012. https://www.chugai-pharm.co.jp/english/ir/rd/technologi es_popup1.html. Accessed 3 Aug 2020.

24. Takeshita Y, Serizawa K, Fujikawa S, et al. The effect of neuromyelitis optica (NMO)-IgG and anti-IL-6 receptor monoclonal antibody (SA237;satralizumab) for barrier function at the blood-brain barrier in vitro [abstract no. P473]. Mult Scler J. 2019;25(Suppl 2):208-9.

25. Lennon-Chrimes S, Baumann HS, Klingelschmitt G, et al. Characterisation of the PK and PD of satralizumab, a recycling antibody, to support Q4W dosing in patients with NMOSD [abstract no. 1483]. Neurology 2020;94(15 Suppl):29.

26. Traboulsee A, Greenberg BM, Bennett JL, et al. Safety and efficacy of satralizumab monotherapy in neuromyelitis optica spectrum disorder: a randomised, double-blind, multicentre, placebocontrolled phase 3 trial. Lancet Neurol. 2020;19(5):402-12.

27. Yamamura T, Kleiter I, Fujihara K, et al. Trial of satralizumab in neuromyelitis optica spectrum disorder. N Engl J Med. 2019;381(22):2114-24.

28. Shimada Y, Munakata Y, Inoo M, et al. Pharmacokinetics, pharmacodynamics, safety, and tolerability of a novel anti-IL-6 receptor recycling antibody SA237: first in-human phase 1 single-and multipledose studies [abstract no. P11197]. Eur J Neurol. 2016;23(Suppl 2):203.

29. Hemingway C, Baumann HS, Kou X, et al. Adolescents with NMOSD achieved similar exposures and favorable safety profile when treated with the adult satralizumab dosing regimen [abstract no. 1492]. Neurology 2020;94(15 Suppl):25.

30. Araki M, Fujihara K, Tsai CP, et al. Efficacy \& safety of satralizumab in subgroup of Asian-region patients with NMOSD in SAkuraSky study [abstract no. O-33-5]. Clinical Neurology. 2019;59(Suppl 1):S245.

31. De Seze J, Weinshenker BG, Terada Y, et al. Efficacy and safety of satralizumab for relapse prevention in neuromyelitis optica spectrum disorder: a pooled analysis from two phase 3 clinical trials [abstract no. P1614]. Mult Scler J. 2019;25(Suppl 2):904-5.

32. Haskova Z, Frishberg B, de Seze J, et al. Efficacy and safety of satralizumab from two phase 3 trials in neuromyelitis optica spectrum disorder [abstract no. 3173]. Invest Ophthalmol Vis Sci. 2020;61:7.

33. Greenberg B, De Seze J, Fox E, et al. Safety of satralizumab based on pooled data from phase 3 studies in patients with neuromyelitis optica spectrum disorder (NMOSD) [abstract no. 1281]. Neurology. 2020;94(15 Suppl):30.

34. Genentech. New longer-term data reinforce safety of Genentechs satralizumab in adults and adolescents with neuromyelitis optica spectrum disorder (NMOSD) [media release]. 22 May 2020. 